\title{
Synthesis and Identification of Some New Metal Complexes of Mixed Schiff Base Ligands
}

\author{
Waleed A. Mahmoud ${ }^{1}$, Hawraa K. Salloom ${ }^{2}$ \\ Department of Chemistry, College of Science of Woman, University of Baghdad, (Iraq)
}

\begin{abstract}
The free Schiff base ligand $\left(H L_{1}\right)$ which was prepared mixed with the Schiff base ligand $\left(H L_{2}\right)$.The product then was reacted with some transition metal salts: $\left(\mathrm{Cu}^{2+}, \mathrm{Ni}^{2+}, \mathrm{Cd}^{2+}, \mathrm{Mn}^{2+}, \mathrm{Zn}^{2+}\right.$ and $\mathrm{Pd}^{2+}$ to get new transition metal complexes. The ligand which were prepared and their transition metal complexes where characterized by physic-chemical spectroscopic techniques such as: FT-IR, UVVis, (C.H.N) spectra, molar conductivity, magnetic moment, mass spectrometer techniques and microanalysis techniques. The results showed the formation and the high purity of the free Schiff base ligands and their metal complexes, indicating that they are in a good agreement with the theoretical values. The fragments of the prepared free Schiff base ligand where identified by mass spectrometer techniques. All analysis of ligands and their metal complexes where in good agreement with the theoretical values which indicated the purity of Schiff base ligands and their metal complexes. From the above data, the molecular structures for all the metal complexes where proposed to be octahedral except Capper and Palladium were square planar.
\end{abstract}

Keywords: Synthesis, identification, transition metal complexes, Mixed ligand, Schiff base.

\section{Introduction}

The branch of inorganic chemistry that deals with the study of coordination compounds is called coordination chemistry (1). The concept of a metal complex has been originated by Alfred Werner who (in 1913) was awarded the first Nobel Prize in inorganic chemistry ${ }^{(2)}$. Whereas the study of a range of coordination compounds is connected to the nature of metal and the ligand $\left({ }^{3)}\right.$. In general, coordination compound is a metal surrounded by neutral molecules or ions called ligands. Ligands are Lewis bases they contain at least one unshared electrons. They are also called complexing agents. Metal ions have empty valance orbitals, they act as Lewis acids ${ }^{(4)}$.

Schiff base In modern coordination chemistry, many different inorganic and organic compounds are used as ligands. But there are two most important classes of ligands in the modern coordination chemistry, namely, azomethines andoximes ${ }^{(5)}$. On the other hand ligands, a metal surrounded by a cluster of ions or molecules are used for preparation of complex compounds named as Schiff's bases ${ }^{(6)}$. Tetra-dentate Schiff's bases are well known for their coordination with various metal ions, forming stable compounds $\left({ }^{7,9)}\right.$. In general Schiff bases (azomethines) have been known since 1864 when Hugo Schiff reported the condensation of primary amines with carbonyl compounds. Now days, the research field dealing with Schiff base coordination chemistry has expanded enormously ${ }^{(10)}$. The common imines are crystalline solids, which are feebly basic but at least some form insoluble salts with strong acids $\left({ }^{11)}\right.$. On the other hand the color of the Schiff bases are due to the presence of this azomethine $(>\mathrm{C}=\mathrm{N})$ linkage and can vary by introducing other auxochromic group ${ }^{(12)}$

\section{Experimental}

\section{A. Instrumentation}

Melting point measurements were carried out at the laboratories of the College of Science for women, University of Baghdad, Ultraviolet- Visible Spectrophotomèter type
(UV-160A Shimadzu) in the region 200-1100 $\mathrm{nm}$ The measurements were carried out at the laboratories of IbnSina Company Baghdad, Iraq, FT.IR Spectra Infrared spectra of the prepared compounds were recorded as potassium bromide disc by using Shimadzu, (8400S) Fourier Transform Infrared Spectrophotometer in the range 4000$400 \mathrm{~cm}^{-1}$. The spectra were carried out at the laboratories of In Ibn-Sina Company Baghdad, Iraq. Element (C.N.H) analysis, have been carried out in (Euro vector model EA3000, single V.3.O single. The measurements were carried out at College of Science, University of Babylon

Molar conductivity Electrical conductivity measurements of the complexes were recorded at $\left(25^{\circ} \mathrm{C} \pm 2{ }^{\circ} \mathrm{C}\right)$ for $\left(1 \times 10^{-3} \mathrm{M}\right)$ $\mathrm{M}$ solution of the samples in DMSO, using a Philips PWdigital conductivity meter. The measurements were carried out at College of Science for women, University of Baghdad, Magnetic measurements. The measurements were carried out at the laboratories of Al-Nahrain University, Baghdad, Iraq.

\section{B. Synthesis of Compounds}

1. Preparationof (Z) 4 (2hydroxybenzylideneamino) -1, 5dimethyl-2-phenyl-1H-pyrazol-3 $(2 \mathrm{H})$-one $\left(\mathrm{L}_{1}\right)$

The Schiff base ligand was prepared by the condensation of the p-amino-2, 3-dimethyl-1-phenyl-3-pyrozoline-5-on (0.5 g, $\quad 0.0247 \mathrm{~mol})$ with the $\left(0.26 \mathrm{~cm}^{3}, \quad 0.0247 \mathrm{~mol}\right)$ salicylaldehyde in methanol $15 \mathrm{~cm}^{3}$. The resulting mixture was then refluxed for $1 \mathrm{~h}$. The yellow solid was collected by filtration, dried at $\left(40^{\circ} \mathrm{C}\right)$ for five hours and then recrystalized from absolute ethanol to give yellow needles, collected by filtration and then dried in oven for four hours at temperature $\left(60^{\circ} \mathrm{C}\right)$, m.p $\left(188-189^{\circ} \mathrm{C}\right)$ and the yield $(86$ $\%)$.

2. Preparation of Schiff base ligand (2- (2- (2-hydroxy benzylideneamino) ethyl) isoindoline-1, 3-dione $\left(\mathrm{L}_{2}\right)$.

A Synthesis of 2- (2-aminoethyl) isoindoline-1, 3-dione: Isobenzofuran-1, 3-dione (0.148 $\mathrm{g}, 1$ mole) was dissolved in

\section{Volume 4 Issue 12, December 2015}




\section{International Journal of Science and Research (IJSR) \\ ISSN (Online) : 2319-7064}

Index Copernicus Value (2013) : 6.14 | Impact Factor (2014) : 5.611

absolute ethanol $\left(15 \quad \mathrm{~cm}^{3}\right)$ with stirring. 2Aminoethanaminium (0.06 g, 1 mole) was dissolved in absolute ethanol $\left(10 \mathrm{~cm}^{3}\right)$ and added to the isobenzofuran-1, 3 -dione solution were mixed in 1:1 mole ratio. The mixture solution was heated under reflux at temperature $\left(40^{\circ} \mathrm{C}\right)$ for $(2$ hours). During this period, a white precipitate was formed. The white solid was collected by filtration and then washed with ethanol $\left(2 \mathrm{~cm}^{3}\right)$ and then dried in oven at temperature $50-70^{\circ} \mathrm{C}$ for two hours, and then kept in desiccator, m.p $\left(123-125^{\circ} \mathrm{C}\right) \&$ the yield $(88 \%)$

2- (2-aminoethyl) isoindoline-1, 3-dione (0.19 g, 1 mole) was dissolved in absolute ethanol $\left(15 \mathrm{~cm}^{3}\right)$ with stirring. Salicyaldehyde $(0.122 \mathrm{~g}, 1$ mole) was dissolved in absolute ethanol $\left(5 \mathrm{~cm}^{3}\right)$, and added to the 2- (2-aminoethyl) isoindoline-1, 3-dione solution were mixed in 1:1 mole ratio. The mixture was heated with stirring under reflux at temperature $\left(40^{\circ} \mathrm{C}\right)$ for 3 hours. During this period the colorless solution turned to the yellow solid. The yellow solid was collected by filtration and then washed with absolute ethanol and then dried in oven for six hours at temperature $\left(60^{\circ} \mathrm{C}\right)$ and then recrystallized from absolute ethanol. The yellow powder collected by filtration and then dried in oven for five hours at temperature $\left(60^{\circ} \mathrm{C}\right)$, m.p (115$\left.117^{\circ} \mathrm{C}\right)$ and the yield $(87 \%)$ The white solid was collected by filtration and then washed with ethanol $\left(2 \mathrm{~cm}^{3}\right)$ and then dried in oven at temperature $50-70^{\circ} \mathrm{C}$ for two hours, and then kept in desiccator, m.p (123-125 $\left.{ }^{\circ} \mathrm{C}\right) \&$ the yield $(88 \%)$

\section{Preparationof $\mathrm{Cu}^{2+}$ Complex}

Schiff base ligand 4- (2-hydroxybenzylideneamino) -1, 5dimethyl-2-phenyl-1H-pyrazol-3 $(2 \mathrm{H})$-one $\left(\mathrm{L}_{1}\right)(1 \mathrm{~g}, 3.253$ mmole) was dissolved in absolute ethanol $\left(20 \mathrm{~cm}^{3}\right)$, Copper (II) chloride (0.437 g, 3.253 mmole) was dissolved in absolute ethanol $\left(15 \mathrm{~cm}^{3}\right)$ an ethanolic solution of $(0.957 \mathrm{~g}$, 3.253 mmole) (2- (2- (2-hydroxy benzylideneamino) ethyl) isoindoline-1, 3-dione $\left(\mathrm{L}_{2}\right)$ was added to the mixture in each case. the solution mixture was refluxed for 4 hours. The mixture was stirred and heated under reflux for two hours at temperature $\left(50-70^{\circ} \mathrm{C}\right)$. During this period, the colour changed for all complexes. The precipitate was collected by filtration, washed with absolute ethanol $\left(10 \mathrm{~cm}^{3}\right)$, and then recrystallized from absolute ethanol. A dark-green were obtained, m.p. $\left(244-246{ }^{\circ} \mathrm{C}\right)$, Yield: $77 \%$.

A similar method as described in section (3) to prepare the other complexes.

\section{Result and Dissection}

\section{FT-IR spectra}

In table (1) The Infrared spectra of the both free Schiff base ligands were measured in the range (4000-400) $\mathrm{cm}^{-1}$, using KBrpellts.

The infrared spectrum of the free Schiff base ligand, 2[2 (2hydroxylbenzylidehydeamino) ethyl] isoindoline-1, 3dione $\left(\mathrm{HL}_{1}\right]$ appeared weak a broad band at $(3433) \mathrm{cm}^{-1}$ was attributed to the stretching vibration of $(\mathrm{OH})$ because of the intra molecular hydrogen bonding $\left({ }^{13)}\right.$.

The band at $(3410) \mathrm{cm}^{-1}$ stretching vibration $(\mathrm{OH})$ of the free Schiff base ligand $\left(\mathrm{HL}_{2}\right)$, the same band appeared (3410) $\mathrm{cm}^{-1}$ in the palladium complex that means no coordinated the oxygen atom of phenolic group to the palladium complex.

The band at (2974) $\mathrm{cm}^{-1}$, (2812) $\mathrm{cm}^{-1}$, (1724) $\mathrm{cm}^{-1}$, (1608) $\mathrm{cm}^{-1}$ and (1546) $\mathrm{cm}^{-1}$ were assigned to the stretching vibration of $v(\mathrm{C}-\mathrm{H})$ aromatic, $\boldsymbol{v}(\mathrm{C}-\mathrm{H})$ aliphatic, $\boldsymbol{v}(\mathrm{C}=\mathrm{O})$, v $(\mathrm{C}=\mathrm{N})$ and $(\mathrm{C}=\mathrm{C})$ respectively. The other bands at (1249) $\mathrm{cm}^{-1}$ was attributed to stretching vibration $v(\mathrm{C}-\mathrm{C}), 1072 \mathrm{~cm}$ ${ }^{1}$ due to $v(\mathrm{C}-\mathrm{O})$

The infrared spectrum of the other free Schiff base ligand, 4(2-hydroxybenzylidene amino) -1, 5-dimethyl-2-phenyl-1Hpyrazol-3 $(2 \mathrm{H})$-one $\left(\mathrm{HL}_{2}\right)$. The weak broad absorption band at $(3410) \mathrm{cm}^{-1}$ due to the stretching vibration of $(\mathrm{OH})$ due to intramolecular hydrogen bonding. The FT-IR spectra of the prepared complexes where compared with that of free ligands $\left(\mathrm{HL}_{1}\right)$ and $\left(\mathrm{HL}_{2}\right)$ in order to determine the coordination sites involved in complexation, that is mean the position of some guid bands in the spectrum of the free ligands where expected to change up on complexation.

The band at $3433 \mathrm{~cm}^{-1}$ and $3410 \mathrm{~cm}^{-1}$ of the free Schiff base ligands, $\left(\mathrm{HL}_{1}\right)$ and $\left(\mathrm{HL}_{2}\right)$ respectively are absent in the $\mathrm{Ni}$ (II), $\mathrm{Cu}$ (II), $\mathrm{Mn}$ (II), and $\mathrm{Zn}$ (II) complexes which is indicated the deprotonation of phenol proton prior to and indicated the coordination of the phenolic group with Nickel Copper, Manganese and Zinc ions. The bands at (3371$3402) \mathrm{cm}^{-1}$ and medium sharp band appeared at (933) $\mathrm{cm}^{-1}$ and (964) $\mathrm{cm}^{-1}$ where assigned to the stretching vibration and rocking of $(\mathrm{OH})$ of water which were indicated the presence of water molecules inside the coordination sphere of the Mn (II), Zn (II), and Cd (II) complexes respectively. The bands at (3394) $\mathrm{cm}^{-1}$ and (3367) $\mathrm{cm}^{-1}$ due to stretching vibration of hydrated water molecule of $\mathrm{Cu}$ (II) complex

The band at $(1685) \mathrm{cm}^{-1}$ which is belong to the carbonyl group of $\left(\mathrm{HL}_{2}\right)$ was shifted to lower frequency in the prepared complexes Nickel, manganese, Zinc and cadmium complexes, which is indicated to coordinated with the above metal ions $\left({ }^{14)}\right.$, The bands of $(\mathrm{C}=\mathrm{N})$ in the free Schiff base ligands, $\left(\mathrm{HL}_{1}\right)$ and $\left(\mathrm{HL}_{2}\right)$ which are observed at $(1608) \mathrm{cm}^{-1}$ and $(1627) \mathrm{cm}^{-1}$ respectively were shifted to lower frequency in all the spectra of the prepared complexes, this indicates the participitationof the nitrogen atom of the azomethine group in coordination. The bands which are observed at rang (1492-1585) $\mathrm{cm}^{-1}$ assigned to the stretching vibration of $(\mathrm{C}=\mathrm{C})$ for all the spectra of metal complexes. The band at (3433) $\mathrm{cm}^{-1}$ in the free Schiff base ligand $\left(\mathrm{HL}_{1}\right)$ showed no changed in the spectrum at Cadmium complex, this indicated uncoordinated the oxygen of phenolic group of the Schiff base ligand $\left(\mathrm{HL}_{1}\right)$

Table 1: FT-IR of free Schiff base ligands and their metal complexes in $\left(\mathrm{cm}^{-1}\right)$ 


\section{International Journal of Science and Research (IJSR) \\ ISSN (Online) : 2319-7064}

Index Copernicus Value (2013) : 6.14 | Impact Factor (2014) : 5.611

\begin{tabular}{|c|c|c|c|c|c|c|c|c|c|c|}
\hline Compounds & v OH & $\begin{array}{c}\text { vC-H } \\
\text { ar }\end{array}$ & vC-H aliph & v $\mathbf{C}=\mathbf{O}$ & $v \mathbf{C}=\mathbf{C}$ & $v \mathbf{C}=\mathbf{N}$ & $\begin{array}{l}\text { vC-O } \\
\text { phen. }\end{array}$ & vM-N & v M-O & $\begin{array}{l}\text { Other } \\
\text { bands }\end{array}$ \\
\hline $\begin{array}{c}\text { Schiff base } \\
\left(\mathrm{HL}_{1}\right)\end{array}$ & 3433 & $\begin{array}{l}2974 \\
2812\end{array}$ & $\begin{array}{l}2708 \\
2576\end{array}$ & 1724 & 1516 & 1606 & 1072 & 540 & 478 & \\
\hline $\begin{array}{l}\text { Schiff base } \\
\left(\mathrm{HL}_{2}\right)\end{array}$ & 3414 & $\begin{array}{l}2993 \\
2954\end{array}$ & $\begin{array}{l}2800 \\
2634\end{array}$ & 1685 & 1558 & 1627 & 1080 & 543 & 493 & - \\
\hline$\left[\mathrm{Mn}\left(\mathrm{L}_{1}\right)\left(\mathrm{L}_{2}\right) \mathrm{H}_{2} \mathrm{O}\right]$ & 3394 & $\begin{array}{l}3055 \\
3020\end{array}$ & 2935 & 1654 & 1539 & $\begin{array}{l}1624 \\
1597\end{array}$ & $\begin{array}{l}1049 \\
1029\end{array}$ & $\begin{array}{l}551 \\
505\end{array}$ & 466 & $\begin{array}{c}\text { Aqu } 933 \\
\mathrm{H}_{2} \mathrm{O}\end{array}$ \\
\hline$\left[\mathrm{Ni}\left(\mathrm{L}_{1}\right)\left(\mathrm{L}_{2}\right) \mathrm{H}_{2} \mathrm{O}\right]$ & 3390 & $\begin{array}{l}3059 \\
3020 \\
\end{array}$ & $\begin{array}{l}2935 \\
2800\end{array}$ & 1654 & 1539 & $\begin{array}{l}1620 \\
1600 \\
\end{array}$ & 1033 & $\begin{array}{l}551 \\
505\end{array}$ & 408 & $\begin{array}{c}\text { Aqu } 933 \\
\mathrm{H}_{2} \mathrm{O}\end{array}$ \\
\hline$\left[\mathrm{Cu}\left(\mathrm{L}_{1}\right)\left(\mathrm{L}_{2}\right)\right] \mathrm{H}_{2} \mathrm{O}$ & 3367 & 3043 & 2954 & $\begin{array}{l}1701 \\
1685 \\
\end{array}$ & 1581 & $\begin{array}{l}1635 \\
1600 \\
\end{array}$ & 1072 & 551 & 420 & $3394 \mathrm{H}_{2} \mathrm{O}$ \\
\hline$\left[\mathrm{Zn}\left(\mathbf{L}_{1}\right)\left(\mathbf{L}_{2}\right) \mathbf{H}_{2} \mathbf{O}\right]$ & 3460 & 3062 & 2939 & 1651 & 1543 & 1604 & $\begin{array}{l}1041 \\
1029\end{array}$ & $\begin{array}{l}524 \\
505\end{array}$ & $\begin{array}{l}455 \\
428\end{array}$ & $\begin{array}{c}\text { Aqu } 933 \\
\mathbf{H}_{2} \mathbf{O}\end{array}$ \\
\hline$\left[\mathrm{Cd}\left(\mathrm{HL}_{1}\right)\left(\mathrm{L}_{2}\right) \mathrm{Cl} . \mathrm{H}_{2} \mathrm{O}\right]$ & - & $\begin{array}{l}3062 \\
2993 \\
\end{array}$ & 2939 & 1651 & 1562 & 1597 & 1141 & 547 & 408 & $\begin{array}{c}\text { Aqu933 } \\
\mathrm{H}_{2} \mathrm{O}\end{array}$ \\
\hline$\left[\operatorname{Pd}\left(\mathrm{L}_{1}\right)\left(\mathrm{HL}_{2}\right) \mathrm{Cl}\right]$ & - & 3059 & $\begin{array}{l}2993 \\
2808\end{array}$ & 1685 & 1562 & $\begin{array}{l}1654 \\
1593\end{array}$ & 1072 & $\begin{array}{l}551 \\
505\end{array}$ & 420 & - \\
\hline
\end{tabular}

\section{Mass Spectrometer}

The mass spectrum of the 2- (2-aminoethyl) isoindoline-1, 3 -dione has been studied at temperature $\left(210^{\circ} \mathrm{C}\right)$. The main high resolution mass spectrometer of this compound is obtained at $210^{\circ} \mathrm{C}$ which is appeared at very clear and higher relative abundance. The molecular ion peak was observed at $\mathrm{m} / \mathrm{z}=190(\mathrm{M})$ (relative abundance is $65 \%$ ) for $\mathrm{C}_{10} \mathrm{H}_{10} \mathrm{O}_{2} \mathrm{~N}_{2}$ which is in excellent agreement with the theoretical value. The other peaks are detected at $(M / Z=194)$ ) (relative abundance is 63\%), corresponding to $[\mathrm{M}$ (190) + $\left.\left(\mathrm{C}_{10} \mathrm{H}_{14} \mathrm{O}_{2} \mathrm{~N}_{2}\right)\right]$, [M (190) - $\left(\mathrm{C}_{8} \mathrm{H}_{5} \mathrm{O}_{2} \mathrm{~N}_{2}\right)$ and [M (165) $\left.\left(\mathrm{C}_{8} \mathrm{H}_{7} \mathrm{ON}_{2}\right)\right]$ respectively. The other peaks which is obtained from the fragmentation of $\mathrm{C}_{10} \mathrm{H}_{14} \mathrm{O}_{2} \mathrm{~N}_{2} \quad(\mathrm{M} / \mathrm{Z}=194)$ are detected at $\mathrm{m} / \mathrm{z}=166$ (relative abundance is $10 \%$ ), 165 (relative abundance is 100\%) and 147 (relative abundance is $10 \%$ ). All the fragmentations can be shown in table (2) Figure (1) and scheme (1).

Table 2: The fragmentation pattern data for the 2- (2aminoethyl) isoindoline-1, 3-dione at $210^{\circ} \mathrm{C}$ Molecular ion

\begin{tabular}{|c|c|c|}
\hline Molecular ion & Relative abundance & Assignment \\
\hline 190 & $65 \%$ & $\mathrm{C}_{10} \mathrm{H}_{10} \mathrm{O}_{2} \mathrm{~N}_{2}$ \\
\hline 165 & $100 \%$ & $\mathrm{C}_{8} \mathrm{H}_{9} \mathrm{O}_{2} \mathrm{~N}_{2}$ \\
\hline 147 & $10 \%$ & $\mathrm{C}_{8} \mathrm{H}_{7} \mathrm{ON}_{2}$ \\
\hline 194 & $63 \%$ & $\mathrm{C}_{10} \mathrm{H}_{14} \mathrm{O}_{2} \mathrm{~N}_{2}$ \\
\hline 194 & $63 \%$ & $\mathrm{C}_{10} \mathrm{H}_{14} \mathrm{O}_{2} \mathrm{~N}_{2}$ \\
\hline 166 & $10 \%$ & $\mathrm{C}_{9} \mathrm{H}_{10} \mathrm{ON}_{2}$ \\
\hline 165 & $100 \%$ & $\mathrm{C}_{9} \mathrm{H}_{9} \mathrm{ON}_{2}$ \\
\hline 147 & $10 \%$ & $\mathrm{C}_{8} \mathrm{H}_{4} \mathrm{ON}_{2}$ \\
\hline
\end{tabular}

The main high resolution mass spectrometer of the free Schiff base ligand, 2-[2-hydroxybenzylideneamino) ethyl] isoindoline-1, 3-dione is carried out at temperature $\left(170^{\circ} \mathrm{C}\right)$ which is illustrated in figure (2), table (3) and Scheme (2). The molecular ion peak for the free Schiff base ligand was showed at $\mathrm{m} / \mathrm{z}=294(\mathrm{M})$ (relative abundance is $33 \%$ ) for $\mathrm{C}_{17}$ $\mathrm{H}_{14} \mathrm{O}_{3} \mathrm{~N}_{2}$ 11which is in a good agreement with the theoretical value. The other molecular ion peaks are detected at $\mathrm{m} / \mathrm{z}=268$ (relative abundance is $100 \%$ ), 194 (relative abundance is $8 \%$ ), 166 (relative abundance is $100 \%$ ), 146 (relative abundance is 63\%), 134 (relative abundance is 134) and 120 (relative abundance is $12 \%$ ) corresponding to[M
(294) - $\left.\left(\mathrm{C}_{16} \mathrm{H}_{14} \mathrm{O}_{3} \mathrm{~N}\right)\right]$, [M (268) $\left.-\mathrm{C}_{10} \mathrm{H}_{14} \mathrm{O}_{2} \mathrm{~N}_{2}\right)$ ], [M (194) $\left(\mathrm{C}_{8} \mathrm{H}_{10} \mathrm{O}_{2} \mathrm{~N}_{2}\right)$ ], [ $\mathrm{M}(166)-\left(\mathrm{C}_{7} \mathrm{H}_{2} \mathrm{O}_{2} \mathrm{~N}_{2}\right)$ ], [M (146) $\left.\left(\mathrm{C}_{5} \mathrm{H}_{2} \mathrm{O}_{2} \mathrm{~N}_{2}\right)\right]$ and [M (134) - $\left(\mathrm{C}_{5} \mathrm{H}_{2} \mathrm{O}_{2} \mathrm{~N}\right)$ ]. OR [M (294) $\left.\left(\mathrm{C}_{16} \mathrm{H}_{14} \mathrm{O}_{3} \mathrm{~N}\right)\right]$, [M (268) - $\left.\left(\mathrm{C}_{12} \mathrm{H}_{6} \mathrm{O}\right)\right]$, [M (166) - $\left(\mathrm{C}_{10} \mathrm{H}_{10} \mathrm{O}\right)$ ], [ $\left.\mathrm{M}(146)-\left(\mathrm{C}_{9} \mathrm{H}_{10} \mathrm{O}\right)\right]$ and $\left[\mathrm{M}(134)-\left(\mathrm{C}_{8} \mathrm{H}_{8} \mathrm{O}\right)\right]$.

Table 3: Fragmentation pattern data for the free Schiff base at temp. $170^{\circ} \mathrm{C}$

\begin{tabular}{|c|c|c|}
\hline Molecular ion $\mathrm{m} / \mathrm{z}$ & Relative abundance & Assignment \\
\hline 294 & $33 \%$ & $\mathrm{C}_{17} \mathrm{H}_{14} \mathrm{O}_{3} \mathrm{~N}_{2}$ \\
\hline 268 & $100 \%$ & $\mathrm{C}_{16} \mathrm{H}_{14} \mathrm{O}_{3} \mathrm{~N}$ \\
\hline 194 & $8 \%$ & $\mathrm{C}_{10} \mathrm{H}_{14} \mathrm{O}_{2} \mathrm{~N}_{2}$ \\
\hline 166 & $100 \%$ & $\mathrm{C}_{8} \mathrm{H}_{10} \mathrm{O}_{2} \mathrm{~N}_{2}$ \\
\hline 146 & $63 \%$ & $\mathrm{C}_{7} \mathrm{H}_{2} \mathrm{O}_{2} \mathrm{~N}_{2}$ \\
\hline 134 & $8 \%$ & $\mathrm{C}_{6} \mathrm{H}_{2} \mathrm{O}_{2} \mathrm{~N}_{2}$ \\
\hline 120 & $12 \%$ & $\mathrm{C}_{6} \mathrm{H}_{2} \mathrm{O}_{2} \mathrm{~N}$ \\
\hline
\end{tabular}

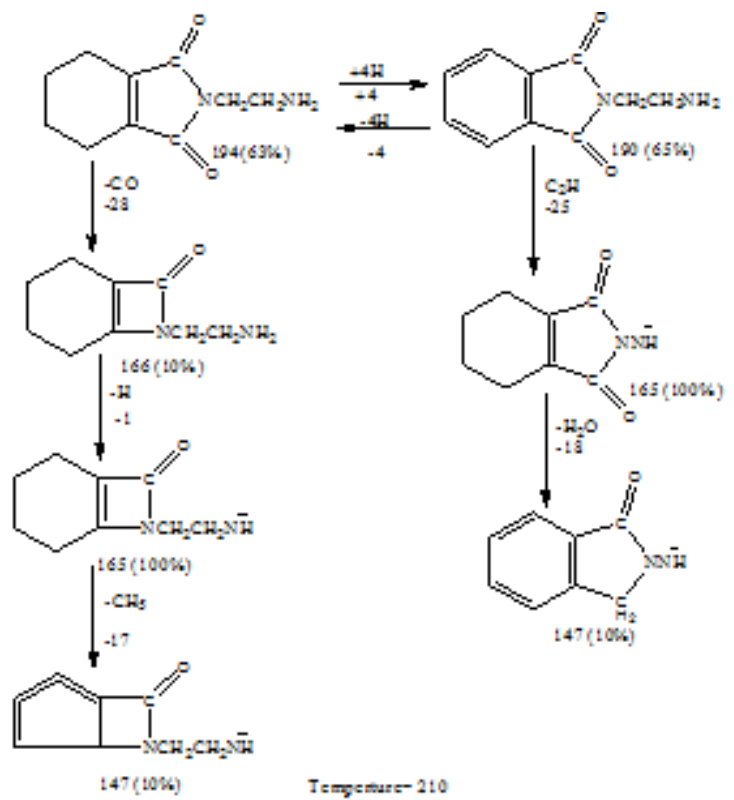

Scheme (1) The fraqmentation pattern of 2- (2- amino ethy 1) iso - indoline - 1,3-doine (Amic acid) at $210 \mathrm{C}^{\circ}$ 


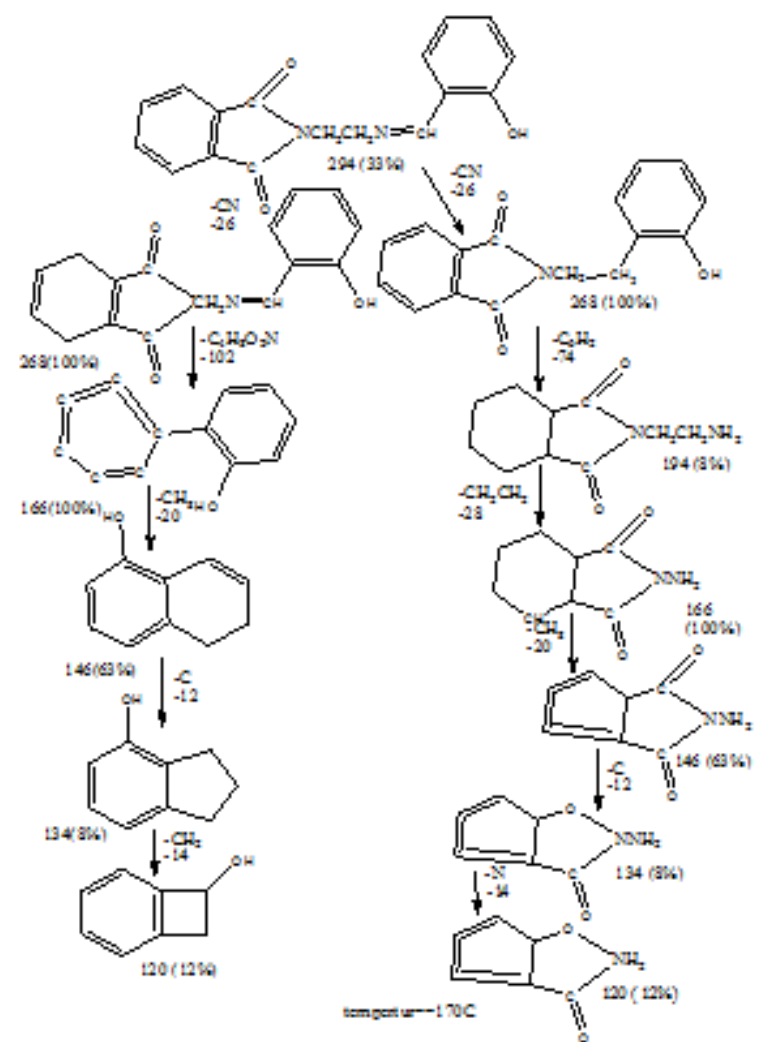

Scheme (2) The fragmentation pattern of Schiff base ligand (2- (2- (2-hydroxy benzylideneamino) ethyl) isoindoline-1, 3dione at $170 \mathrm{C}^{\circ}$

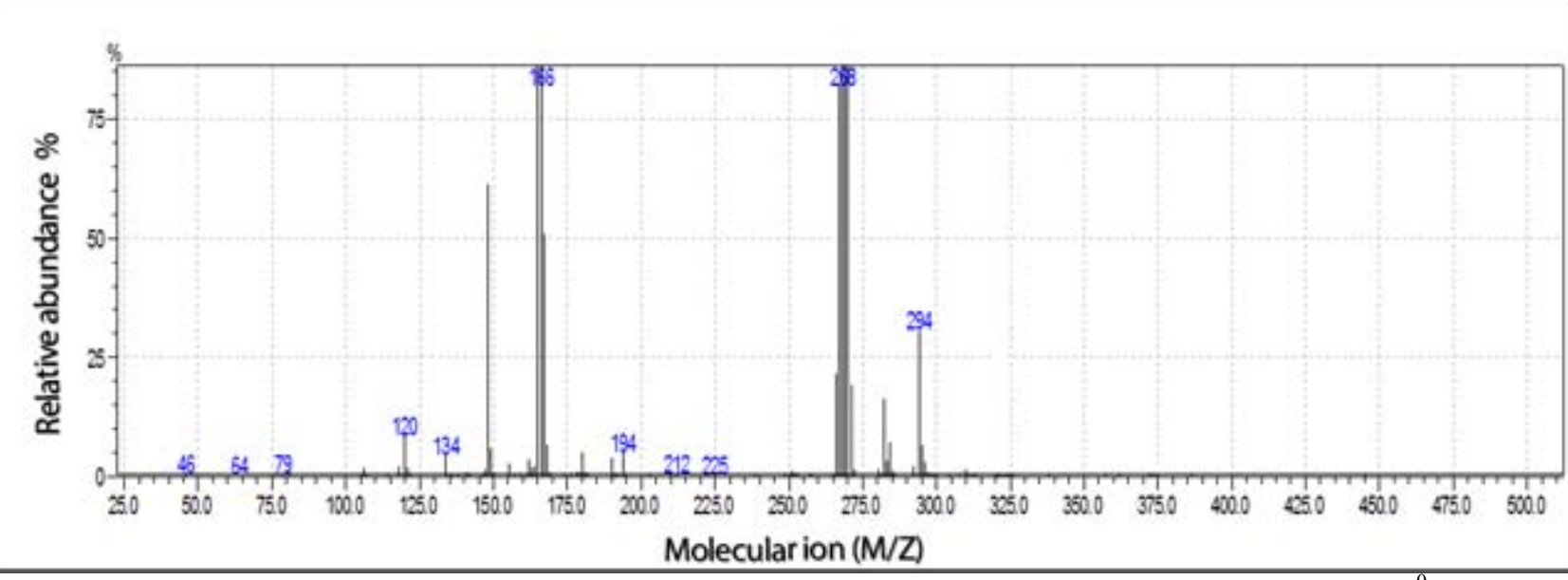

Figure 1: Mass spectrum of Schiff base from 2- (2- amino ethyl) iso - indoline $-1,3-$ doine at $210^{\circ} \mathrm{C}$

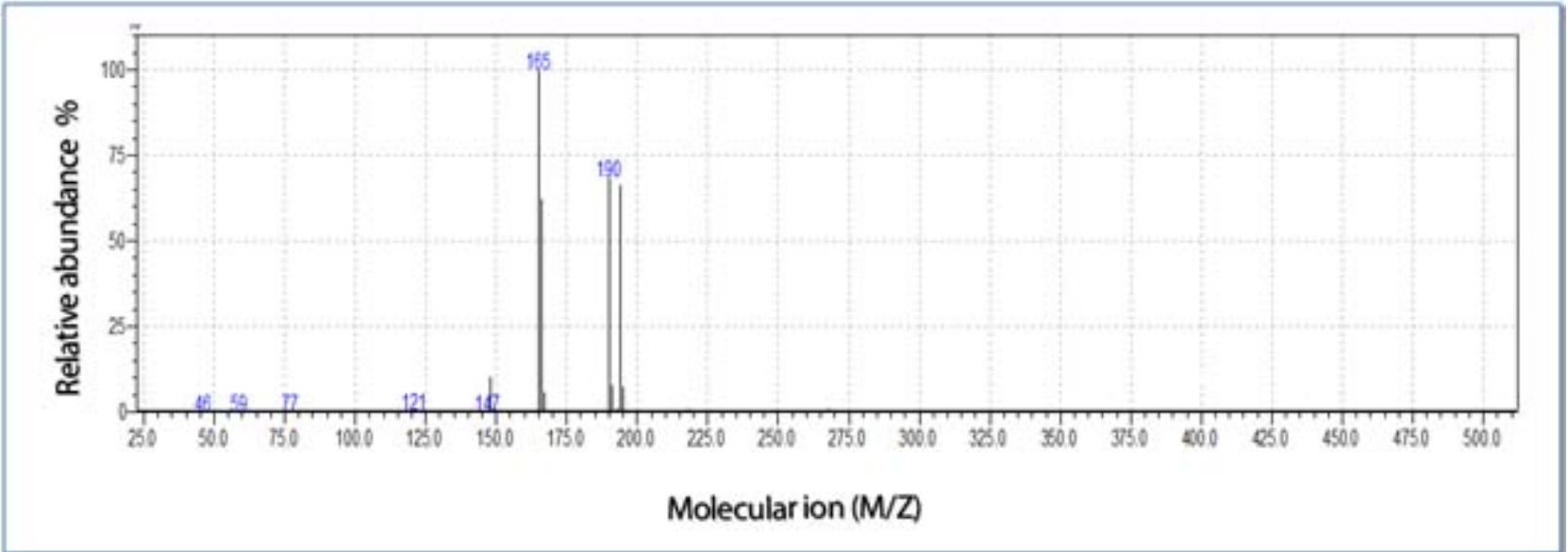

Figure 2: Mass spectrum of Schiff base $\left(\mathrm{HL}_{1}\right)$ at temp. $170^{\circ} \mathrm{C}$.

Volume 4 Issue 12, December 2015 


\section{International Journal of Science and Research (IJSR) \\ ISSN (Online) : 2319-7064}

Index Copernicus Value (2013) : 6.14 | Impact Factor (2014) : 5.611

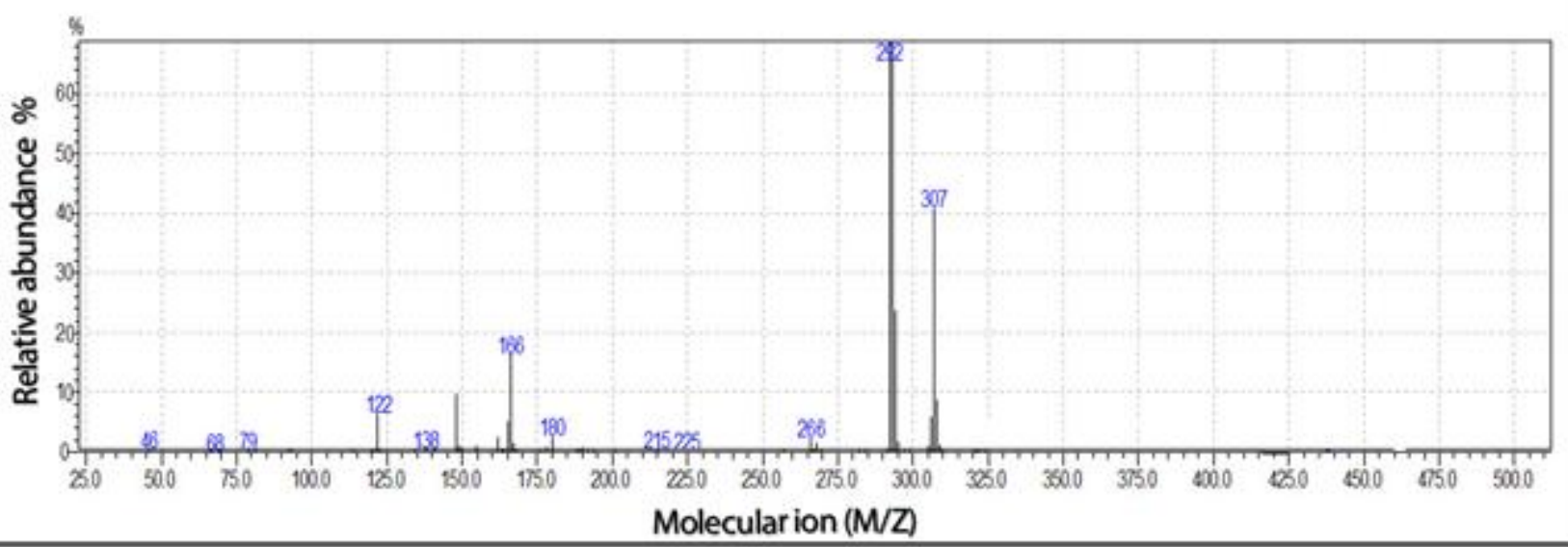

Figure 3: Mass spectrum of Schiff base $\left(\mathrm{HL}_{2}\right)$ from at $210{ }^{\circ} \mathrm{C}$.

Table 4: Some analytical \& physical properties for the Schiff base ligands and their metalcomplexes.

\begin{tabular}{|c|c|c|c|c|c|c|c|c|c|c|c|c|c|c|}
\hline \multirow{2}{*}{ Compounds } & \multirow{2}{*}{ M.w.t } & \multirow{2}{*}{$\begin{array}{l}\text { Molecular } \\
\text { formula }\end{array}$} & \multirow[b]{2}{*}{ Color } & \multirow{2}{*}{ Yield\% } & \multirow{2}{*}{ m.p. ${ }^{\circ} \mathrm{C}$} & \multicolumn{3}{|c|}{$\begin{array}{c}\text { Microanalysis (Calc.) } \\
\text { Exp. }\end{array}$} & \multirow{2}{*}{$\begin{array}{l}\text { M \% } \\
\text { (Calc.) } \\
\text { Exp }\end{array}$} & \multirow[b]{2}{*}{$\mathrm{Cl} \%$} & \multirow{2}{*}{$\begin{array}{c}\mathrm{SO}_{4} \\
\%\end{array}$} & \multirow{2}{*}{$\begin{array}{c}\text { Molar } \\
\text { Conductance } \\
\left(\mu \mathrm{s} / \mathrm{cm}^{-1}\right)\end{array}$} & \multirow{2}{*}{$\begin{array}{l}\mu \text { eff. } \\
\text { (B.M.) }\end{array}$} & \multirow[b]{2}{*}{$\begin{array}{l}\text { Ratio } \\
\text { M/L }\end{array}$} \\
\hline & & & & & & \begin{tabular}{|l|}
$\mathrm{C}$ \\
$\%$
\end{tabular} & $\begin{array}{l}\mathrm{H} \\
\%\end{array}$ & $\begin{array}{l}\mathbf{N} \\
\%\end{array}$ & & & & & & \\
\hline Schiff base $\left(\mathrm{HL}_{1}\right)$ & 294.30 & $\mathrm{C}_{17} \mathrm{H}_{14} \mathrm{~N}_{2} \mathrm{O}_{3}$ & Yellow & 77 & $\begin{array}{l}115- \\
117\end{array}$ & $\begin{array}{l}(69.34) \\
69.55\end{array}$ & $\begin{array}{c}(4.76) \\
4.77\end{array}$ & $\begin{array}{l}(9.52) \\
10.05\end{array}$ & - & - & - & - & - & $1: 1: 1$ \\
\hline Schiff base $\left(\mathrm{HL}_{2}\right)$ & & $\mathrm{C}_{18} \mathrm{H}_{17} \mathrm{~N}_{3} \mathrm{O}_{2}$ & Yellow & 70 & $\begin{array}{l}199- \\
200\end{array}$ & $\begin{array}{l}(70.36) \\
71.14\end{array}$ & $\begin{array}{c}(5.54) \\
5.33\end{array}$ & $\begin{array}{l}(13.68) \\
13.0\end{array}$ & - & - & - & - & - & $1: 1: 1$ \\
\hline$\left[\mathrm{pd}\left(\mathrm{L}_{1}\right)\left(\mathrm{HL}_{2}\right) \mathrm{Cl}\right]$ & 741 & $\begin{array}{c}\mathrm{C}_{35} \mathrm{H}_{30} \\
\mathrm{~N}_{5} \mathrm{O}_{5} \mathrm{ClPd}\end{array}$ & $\begin{array}{l}\text { Yellwish- } \\
\text { green }\end{array}$ & 61 & $\begin{array}{l}135- \\
137\end{array}$ & $\begin{array}{l}(56.68) \\
57.01\end{array}$ & $\left(\begin{array}{c}(4.05) \\
4.11\end{array}\right.$ & $\begin{array}{l}(9.45) \\
10.07\end{array}$ & $\begin{array}{l}(14.3) \\
14.05\end{array}$ & $\operatorname{Pd} 4.72$ & - & $\begin{array}{c}19 \\
\text { Non - } \\
\text { electrolyte }\end{array}$ & Zero & $1: 1: 1$ \\
\hline$\left[\mathrm{Cd}\left(\mathrm{HL}_{1}\right)\left(\mathrm{L}_{2}\right) \mathrm{Cl}\right]$ & 765 & $\mathrm{C}_{35} \mathrm{H}_{31} \mathrm{~N}_{5} \mathrm{O}_{6} \mathrm{ClCd}$ & Yellow & 81 & $\begin{array}{l}195- \\
197\end{array}$ & $\begin{array}{l}(54.9) \\
55.11\end{array}$ & $\begin{array}{c}(4.18) \\
4.56\end{array}$ & $\begin{array}{l}(9.15) \\
10.01\end{array}$ & $\begin{array}{l}(14.64) \\
15.03\end{array}$ & $\mathrm{Cd} 4.57$ & - & $\begin{array}{c}12 \\
\text { Non- } \\
\text { electrolyte }\end{array}$ & zero & $1: 1: 1$ \\
\hline$\left[\mathrm{Mn}\left(\mathrm{L}_{1}\right)\left(\mathrm{L}_{2}\right) \mathrm{Cl}\right]$ & 672 & $\mathrm{C}_{35} \mathrm{H}_{31} \mathrm{~N}_{5} \mathrm{O}_{6} \mathrm{Mn}$ & Dark-Brown & 65 & $\begin{array}{l}281- \\
283\end{array}$ & $\begin{array}{l}(62.5) \\
63.03\end{array}$ & $\begin{array}{c}(4.61) \\
5.00\end{array}$ & $\begin{array}{l}(10.4) \\
10.51\end{array}$ & $\begin{array}{c}(8.184) \\
8.4\end{array}$ & Mns - & - & $\begin{array}{c}17 \\
\text { Non - } \\
\text { electrolyte }\end{array}$ & 5.05 & $1: 1: 1$ \\
\hline $\begin{array}{c}{\left[\mathrm{Zn}\left(\mathrm{L}_{1}\right)\left(\mathrm{L}_{2}\right)\left(\mathrm{H}_{2} \mathrm{O}\right)\right.} \\
]\end{array}$ & 682 & $\mathrm{C}_{35} \mathrm{H}_{31} \mathrm{~N}_{4} \mathrm{O}_{6} \mathrm{Zn}$ & Off-white & 78 & $135 \mathrm{~d}$ & $(61.58)$ & (4.45) & (10.26) & $(9.33)$ & - & - & $\begin{array}{c}20 \text { non- } \\
\text { electrolyte }\end{array}$ & Zero & 1:1:1 \\
\hline $\begin{array}{c}{\left[\mathrm{Ni}\left(\mathrm{L}_{1}\right)\left(\mathrm{L}_{2}\right)\left(\mathrm{H}_{2} \mathrm{O}\right)\right.} \\
]\end{array}$ & 1675.7 & $\mathrm{C}_{35} \mathrm{H}_{31} \mathrm{~N}_{5} \mathrm{O}_{6} \mathrm{Ni}$ & Orange & 77 & $\begin{array}{l}192- \\
194\end{array}$ & $\begin{array}{l}(62.15) \\
63.16\end{array}$ & $\begin{array}{l}(4.6) \\
5.03\end{array}$ & $\begin{array}{l}(10.36) \\
10.58\end{array}$ & $\begin{array}{l}(8.68) \\
9.04\end{array}$ & $\begin{array}{l}- \\
-\end{array}$ & $\begin{array}{l}- \\
-\end{array}$ & $\begin{array}{c}20 \\
\text { Non- } \\
\text { electrolyte }\end{array}$ & 3.04 & $1: 1: 1$ \\
\hline$\left[\mathrm{Cu}\left(\mathrm{L}_{1}\right)\left(\mathrm{L}_{2}\right)\right] \mathrm{H}_{2} \mathrm{O}$ & 680.45 & $\mathrm{C}_{35} \mathrm{H}_{31} \mathrm{~N}_{5} \mathrm{O}_{6} \mathrm{Cu}$ & Dark-green & 77 & 290d & $\begin{array}{l}(61.72) \\
62.12\end{array}$ & $\begin{array}{c}(4.55) \\
4.64\end{array}$ & $\begin{array}{l}(10.3) \\
9.98\end{array}$ & \begin{tabular}{|c|}
6.32 \\
10.01
\end{tabular} & $\begin{array}{l}- \\
-\end{array}$ & $\begin{array}{l}- \\
-\end{array}$ & $\begin{array}{c}18 \\
\text { Non- } \\
\text { electrolyte }\end{array}$ & 1.61 & $1: 1: 1$ \\
\hline
\end{tabular}

Electronic absorption spectra of the 2- (2-aminoethyl) isoindoline-1, 3-dione and Schiff base ligands

The electronic absorption spectra in the ultraviolet and visible region of the spectrum are characterize by the presence of several sharp bands of relatively high intensity and high molar absorptive the weak broad absorption band which are appeared in the visible region of the spectrum with low absorption intensity and low molar extinction coefficient belong to the transition of the metal ions within the $(\mathrm{d}-\mathrm{d})$ electronic transition $\left({ }^{15)}\right.$.

The visible electronic spectra of the prepared metal complexes should lend themselves most easily to theoretical interpretation because of the presence of unpaired electrons in the $\mathrm{d}$ - orbital.

The color of the prepared transition metal complexes are different from the color of their metal salts and ligands, this is good indication of coordination of the central metal ion through the donor atoms of their ligands, therefore the color of the prepared transition metal showed different characterization and positions $\left({ }^{16-17)}\right.$ electronic spectra of 2(2- aminoethyl) isoindoline- 1, 3- Dione. The electronic spectra of 2- (2-Aminoethyl) isoindoline - 1, 3-dione the free Schiff base ligands and their metal complexes were carried out in the range (200-1100) $\mathrm{nm}$ in dimethyl sulphoxide (DMSO) as solvent at room temperature.

The electronic spectrum of 2- (2-aminoethyl) isoindoline appeared one absorption band at $(275 \mathrm{~nm})$ can be assigned to $\left(\pi-\pi^{*}\right)$ electronic transition of the carbonyl group $(\mathrm{C}=0)$ as shown in figure $(4)(18,19)$.

The spectrum of the free Schiff base ligand $\left(\mathrm{HL}_{1}\right)$ showed two absorption bands, the first band at $\left(266 \mathrm{~nm}, 37593 \mathrm{~cm}^{-1}\right)$ attributed to $\left(\pi-\pi^{*}\right)$ electronic transition, the second band at $\left(316 \mathrm{~nm}, 31645 \mathrm{~cm}^{-1}\right)$ assigned to $\left(\mathrm{n}-\pi^{*}\right)$ electronic transition of azomethine group as illustrated in figure (5).

The spectrum of the second free Schiff base ligand $\left(\mathrm{HL}_{2}\right)$ exhibited two absorption bands, the first band at $(271 \mathrm{~nm}$, $\left.36900 \mathrm{~cm}^{-1}\right)$ due to $\left(\pi-\pi^{*}\right)$ electronic transition.while

\section{Volume 4 Issue 12, December 2015}




\section{International Journal of Science and Research (IJSR) \\ ISSN (Online) : 2319-7064}

Index Copernicus Value (2013) : 6.14 | Impact Factor (2014) : 5.611

thesecond band at $\left(344 \mathrm{~nm}, 29069 \mathrm{~cm}^{-1}\right)$ attributed to $\left(\mathrm{n}-\pi^{*}\right)$ electronic transition of azomethine group as shown in figure (6) $(16,17)$

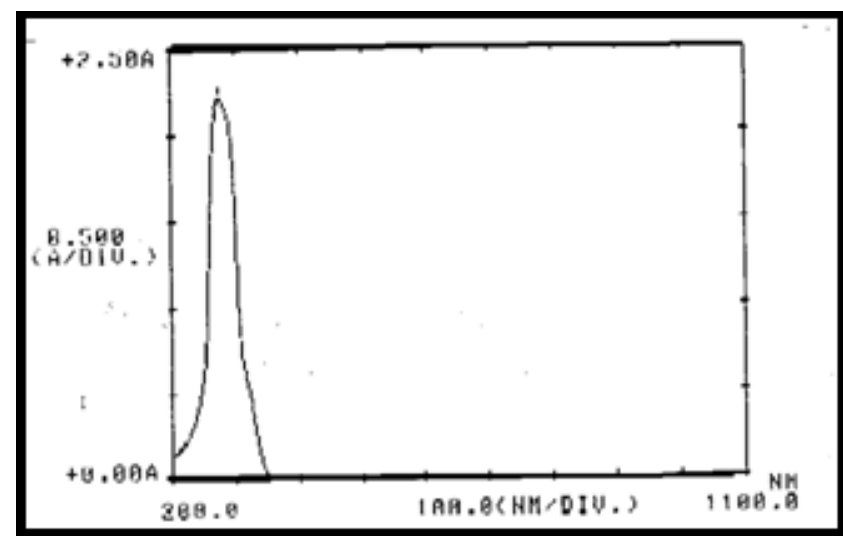

Figure 4: Electronic spectrum of 2- (2-aminoethyl) isoindoline

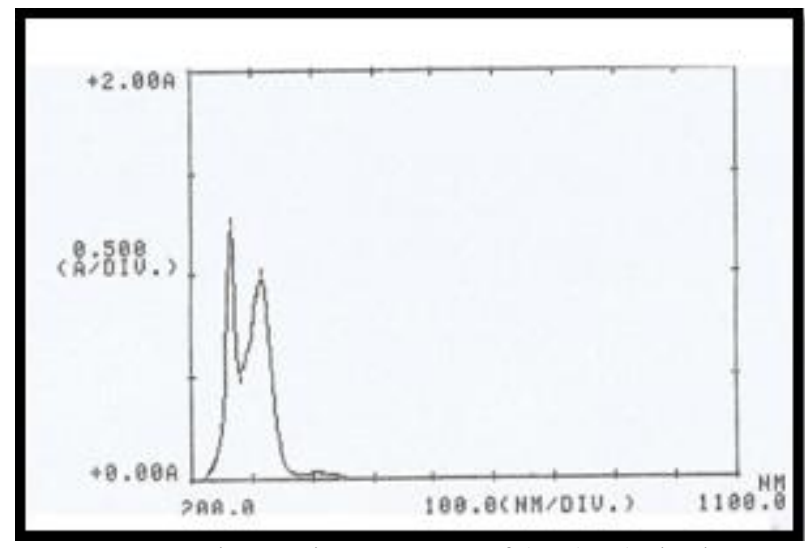

Figure 5: Electronic spectrum of (2- (2- (2-hydroxy

benzylideneamino) ethyl) isoindoline-1, 3-dione ( $\left.\mathrm{HL}_{1}\right)$

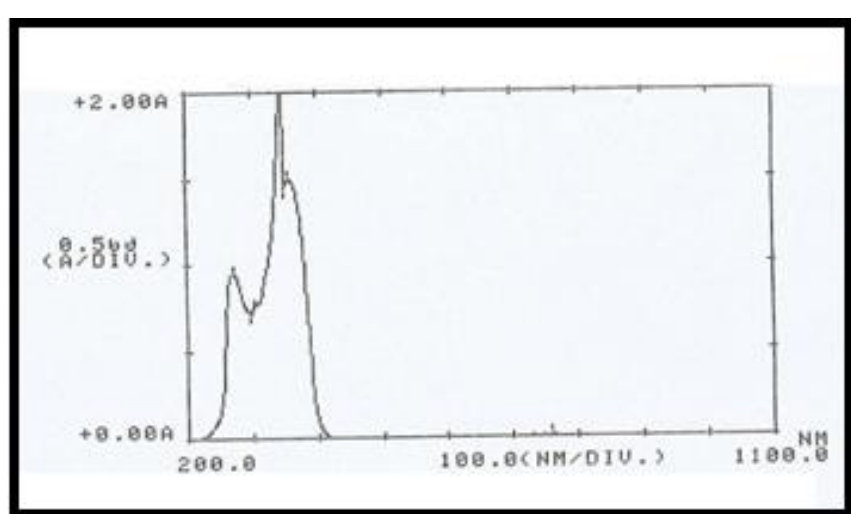

Figure 6: Electronic spectrum of4- (2-

hydroxybenzylideneamino) -1, 5-dimethyl-2-phenyl-HPyrazole-3 (2H) -one (HL2)

\section{Electronic spectra of the prepared metal complexes}

The spectrum of the nickel complex, [ Ni $\left.\left(\mathrm{L}_{1}\right)\left(\mathrm{L}_{2}\right)\left(\mathrm{H}_{2} \mathrm{O}\right)\right]$, in figure (7) exhibited five absorption bands, the first absorption band at $\left(268 \mathrm{~nm}, 37313 \mathrm{~cm}^{-1}\right)$ attributed to $\left(\pi-\pi^{*}\right)$ electronic transition, the second absorption bands at $(345 \mathrm{~nm}$, $\left.28985 \mathrm{~cm}^{-1}\right)$ attributed to $\left(\mathrm{n}-\pi^{*}\right)$ electronic transition, the other three absorption bands appeared at $\left(416 \mathrm{~nm}, 24038 \mathrm{~cm}^{-}\right.$ $\left.{ }^{1}\right),\left(453 \mathrm{~nm}, 22075 \mathrm{~cm}^{-1}\right)$ and $\left(763 \mathrm{~nm}, 13698 \mathrm{~cm}^{-1}\right)$ can be assigned to charge transfer (C.T.), $\left({ }^{3} \mathrm{~A}_{2} \mathrm{~g}(\mathrm{~F}) \rightarrow{ }^{3} \mathrm{~T}_{1} \mathrm{~g}(\mathrm{p})\right)$ and $\left({ }^{3} \mathrm{~A}_{2} \mathrm{~g}(\mathrm{~F}) \rightarrow{ }^{3} \mathrm{~T}_{1} \mathrm{~g}(\mathrm{~F})\right)$ electronic transitions respectively,
These electronic transitions of the complex are characterized to the octahedral geometry around the nickel ion, the value of magnetic effective is 3.04 B.M. which confirmed the suggested structure is octahedral geometry around to nicked ion $(20,21)$.

The spectrum of copper complex, $\left[\mathrm{Cu}\left(\mathrm{L}_{1}\right)\left(\mathrm{L}_{2}\right)\right] \mathrm{H}_{2} \mathrm{O}$, showed four absorption peak, the first peak, the first peak is high intensity and high molar extinction coefficient displayed at $\left(272 \mathrm{~nm}, 36764 \mathrm{~cm}^{-1}\right)$ attributed to $\left(\pi-\pi^{*}\right)$ electronic, the second peak at $\left(357 \mathrm{~nm}, 28011 \mathrm{~cm}^{-1}\right)$ assigned to $\left(n-\pi^{*}\right)$ electronic transition, the third peak at $(492 n m$, $\left.20408 \mathrm{~cm}^{-1}\right)$ attributed to (d-d) transition type $\left({ }^{2} \mathrm{~B}_{1} \mathrm{~g} \rightarrow{ }^{1} \mathrm{Eg}\right)$ and the fourth peak appeared low intensity absorption and low molar extinction coefficient is observed at $(998 \mathrm{~nm}$, $\left.10020 \mathrm{~cm}^{-1}\right)$ can be assigned $\left({ }^{2} \mathrm{~B}_{1} \mathrm{~g} \rightarrow{ }^{2} \mathrm{~A}_{2} \mathrm{~g}\right)$ electronic transition. From these electronic transitions which is suggested the copper complex is octahedral geometry around the copper ion $\left({ }^{22,23)}\right.$ which is illustrated in figure (8).

The spectrum of manganese complex, $\left[\mathrm{Mn}\left(\mathrm{L}_{1}\right)\left(\mathrm{L}_{2}\right) \mathrm{H}_{2} \mathrm{O}\right.$ ], which is illustrated in figure (9) exhibited four absorption bands, the first second and third are high intensity absorption and high molar extinction coefficient are appeared at (274 $\left.\mathrm{nm}, 36496 \mathrm{~cm}^{-1}\right)$ and $\left(345 \mathrm{~nm}, 28985 \mathrm{~cm}^{-1}\right)$ and $(357 \mathrm{~nm}$, $\left.28011 \mathrm{~cm}^{-1}\right)$ assigned to $\left(\pi-\pi^{*}\right),\left(\mathrm{n}-\pi^{*}\right)$ and charge transfer (C.T.) with in $\left({ }^{6} \mathrm{~A}_{1} \mathrm{~g} \rightarrow{ }^{4} \mathrm{~A}_{1} \mathrm{~g},{ }^{4} \mathrm{E}\right.$ (g) ) electronic transitions, we can suggested the manganese complex is octahedral geometry around the manganese ion $\left({ }^{24-27)}\right.$.

The spectra of Zinc and Cadmium complexes [ $\mathrm{Zn}\left(\mathrm{L}_{1}\right)\left(\mathrm{L}_{2}\right)$ $\mathrm{H} 2 \mathrm{O}]$, and [Cd $\left.\left(\mathrm{L}_{1}\right)\left(\mathrm{L}_{2}\right)\left(\mathrm{H}_{2} \mathrm{O}\right)\right]$ showed three absorption bands, the first bands, the first bands at $(266 \mathrm{~nm}, 37594 \mathrm{~cm}$ $\left.{ }^{1}\right)$, and $\left(267 \mathrm{~nm}, 37453 \mathrm{~cm}^{-1}\right)$ due to $\left(\pi-\pi^{*}\right)$ the electronic transitions, the other absorption bands are high intensity absorption and high molar extinction coefficients appeared at $\left(245 \mathrm{~nm}, 28985 \mathrm{~cm}^{-1}\right)$ and $\left(357 \mathrm{~nm}, 28011 \mathrm{~cm}^{-1}\right)$ attributed to $\left(\mathrm{n}-\pi^{*}\right)$ and charge transfer (C.T.) electronic transitions respectively. The absence of the absorption bands in the visible range in the electronic spectra of Zinc, and Cadmium complexes indicated no (d-d) electronic transitions happened because of the electronic configuration of the above metal and d-system. The value of magnetic moment of these metal ion were found to be 0.0B.M (diamagnetic) which are in a goad agreement with theoretical values of the magnetic moment and form the corresponding electronic transition which are confirmed these complexes are octahedral around Zinc, and Cadmium ions ${ }^{28-30)}$ as shown in figure (10) (11).

The spectrum of Palladium complex, [pd $\left.\left(\mathrm{L}_{1}\right)\left(\mathrm{L}_{2}\right)\right] \mathrm{H}_{2} \mathrm{O}$ as shown in figure (12) gave absorption bands, the first sharp band appeared five at $\left(265 \mathrm{~nm}, 37736^{\mathrm{cm}^{-1}}\right)$ attributed to $(\pi$ $\left.\pi^{*}\right)$ electronic transition the second band at $(357 \mathrm{~nm}$, $\left.28011 \mathrm{~cm}^{-1}\right)$ assigned to $\left(\mathrm{n}-\pi^{*}\right)$ electronic transition the third absorption band is high intensity absorption and high molar extinction coefficient observed at $\left(380 \mathrm{~nm}, 26315 \mathrm{~cm}^{-1}\right)$ due to the charge transfer (C.T.) and the four the absorption band is low intensity and low molar absorptivity appeared at (694, $\left.14409 \mathrm{~cm}^{-1}\right)$ can be assigned to $\left({ }^{1} \mathrm{~A}_{1} \mathrm{~g}{ }^{1} \mathrm{~B}_{1} \mathrm{~g}\right)$ electronic transition, the last absorption band at $\left(794 \mathrm{~nm}, 12594 \mathrm{~cm}^{-1}\right)$ attributed to $\left({ }^{1} \mathrm{~A}_{1} \mathrm{~g}{ }^{1} \mathrm{~A}_{2} \mathrm{~g}\right)$ electronic the magnetic value is 0.0B.M. (diamagnetic) which in a good agreement with the

\section{Volume 4 Issue 12, December 2015}




\section{International Journal of Science and Research (IJSR) \\ ISSN (Online) : 2319-7064}

Index Copernicus Value (2013) : 6.14 | Impact Factor (2014) : 5.611

theoretical value. Know the value of magnetic effective and electronic transition indicated the palladium complex is square planar geometry around the Palladium ion $\left.{ }^{(31-33)}\right)$. The suggested geometry of the complexes was demonstrated at figures (13-18).

Table (5) the electronic spectra of 2-(2-aminoethyl)isiindoline-1,3dione, free ligands and their metal complexes (conc. $1 \times 10^{-3}$ mole /l).

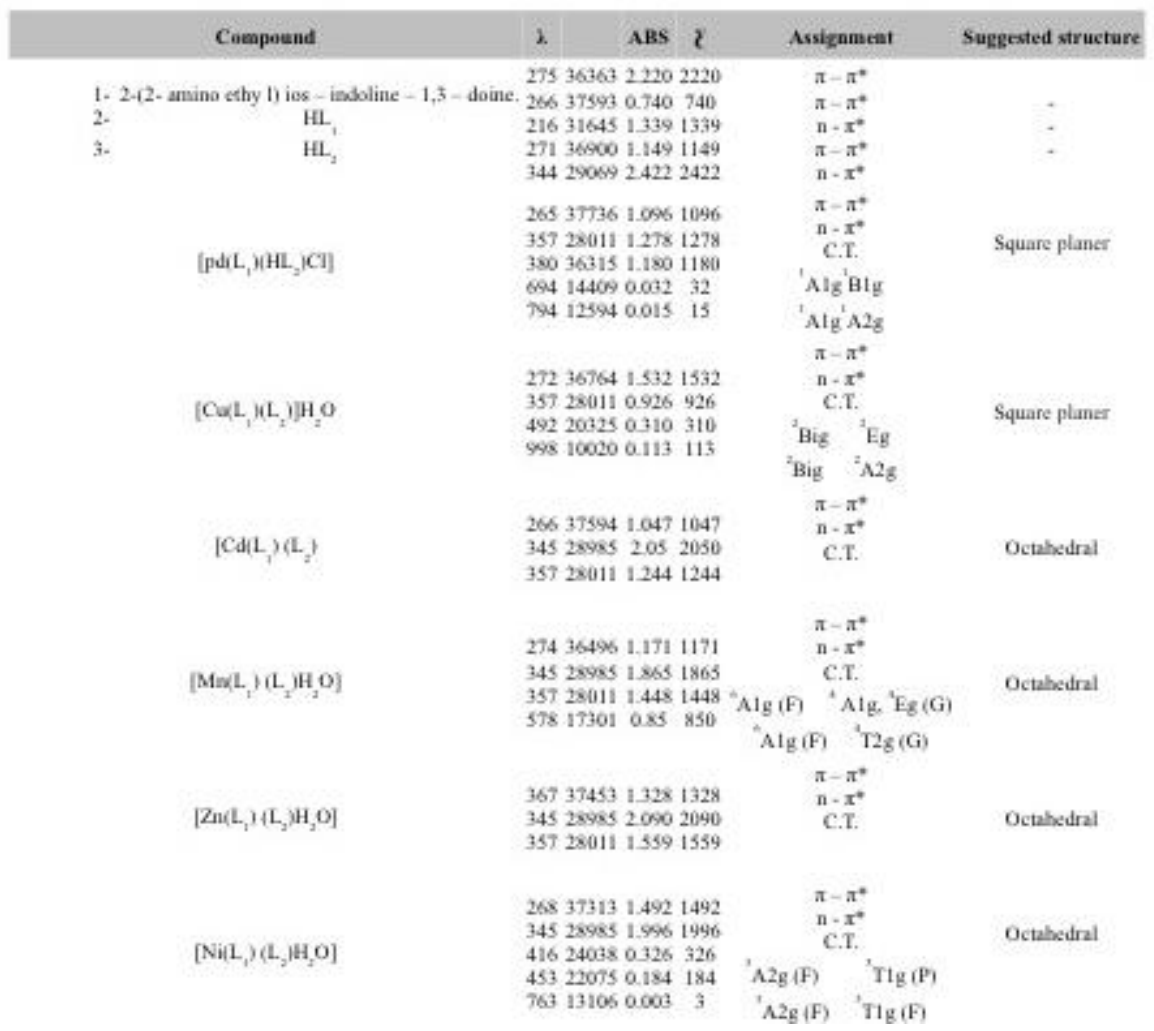

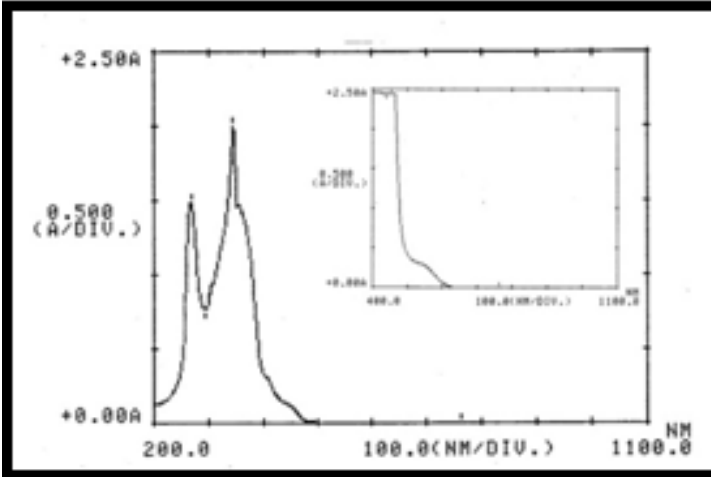

Figure 7: Electronic spectrum of, $\left[\mathrm{Ni}\left(\mathrm{L}_{1}\right)\left(\mathrm{L}_{2}\right)\left(\mathrm{H}_{2} \mathrm{O}\right)\right.$ ]complex

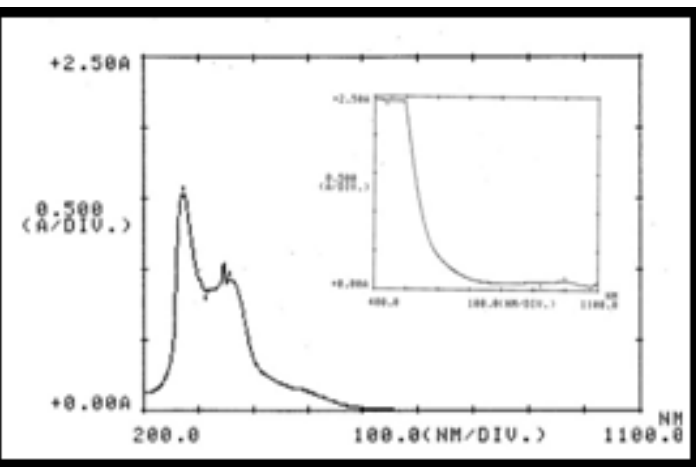

Figure 8: Electronic spectrum of $\left.\left[\mathrm{Cu}\left(\mathrm{L}_{1}\right)\left(\mathrm{L}_{2}\right)\right] \mathrm{H}_{2} \mathrm{O}\right)$ ]complex

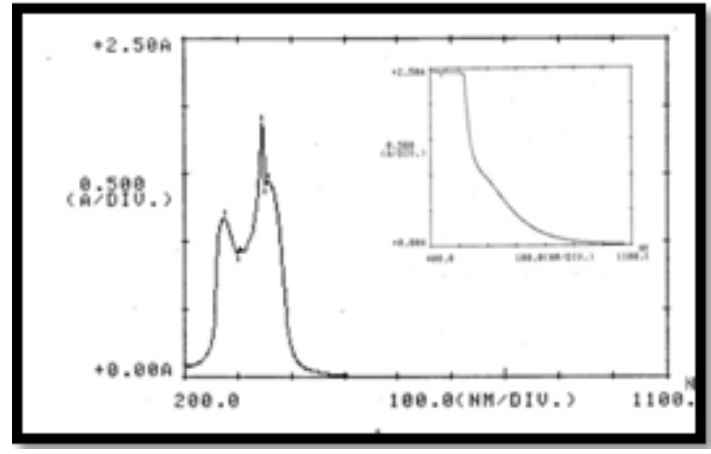

Figure 9: Electronic spectrum of[Mn $\left.\left(\mathrm{L}_{1}\right)\left(\mathrm{L}_{2}\right) \mathrm{H}_{2} \mathrm{O}\right]$ complex

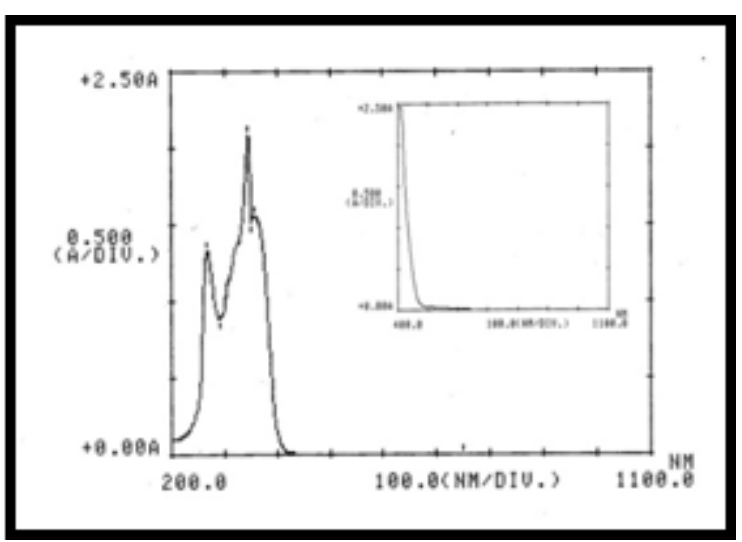

Volume 4 Issue 12, December 2015 
Figure 10: Electronic spectrum of $\left[\mathrm{Zn}\left(\mathrm{L}_{1}\right)\left(\mathrm{L}_{2}\right) \mathrm{H} 2 \mathrm{O}\right]$ complex

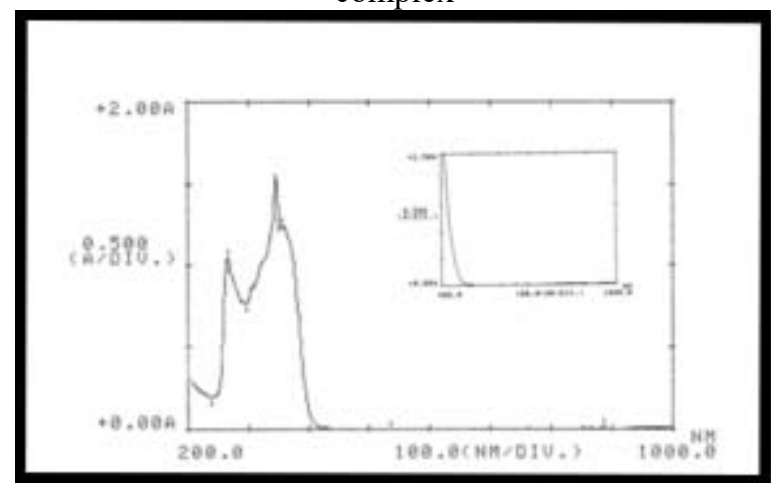

Figure 11: Electronic spectrum of[Cd (L1) (L2) (H2O) ] complex

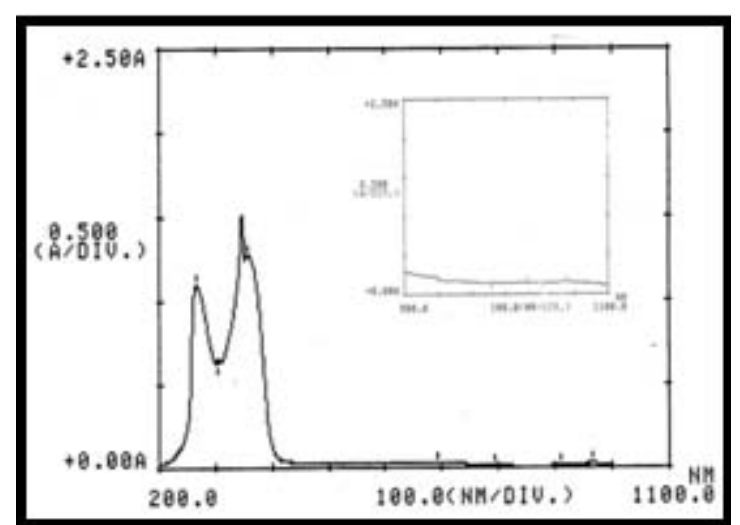

Figure 12: Electronic spectrum of $\left[\mathrm{pd}\left(\mathrm{L}_{1}\right)\left(\mathrm{L}_{2}\right)\right] \mathrm{H} 2 \mathrm{O}$ complex

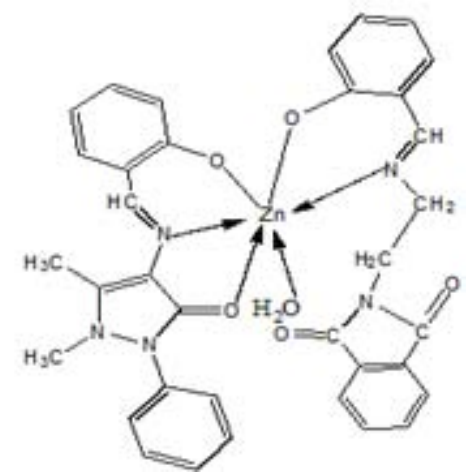

Figure 13: Suggested the structure of $\left[\mathrm{Zn}\left(\mathrm{L}_{1}\right)\left(\mathrm{L}_{2}\right) \mathrm{H}_{2 \mathrm{O}}\right]$

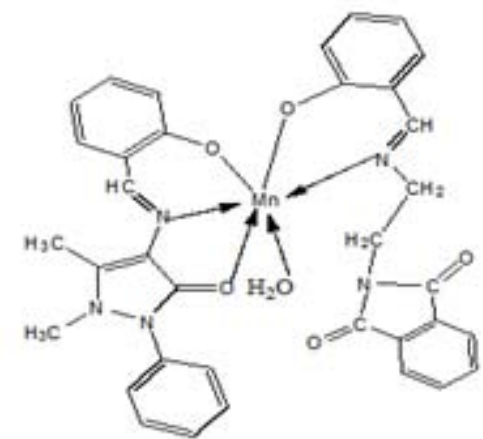

Figure 14: Suggested the structure of[Mn $\left.\left(\mathrm{L}_{1}\right)\left(\mathrm{L}_{2}\right) \mathrm{H}_{2} \mathrm{O}\right]$

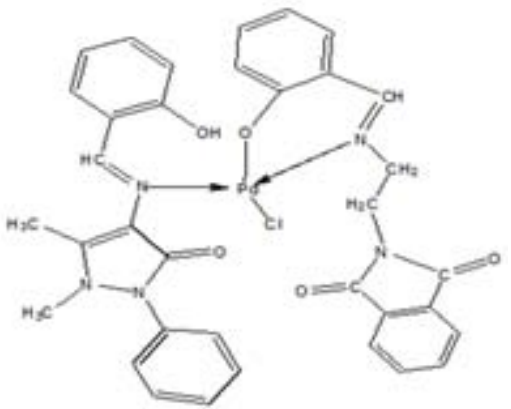

Figure 15: Suggested the structure of $\left[p d\left(\mathrm{~L}_{1}\right)\left(\mathrm{HL}_{2}\right) \mathrm{Cl}\right]$

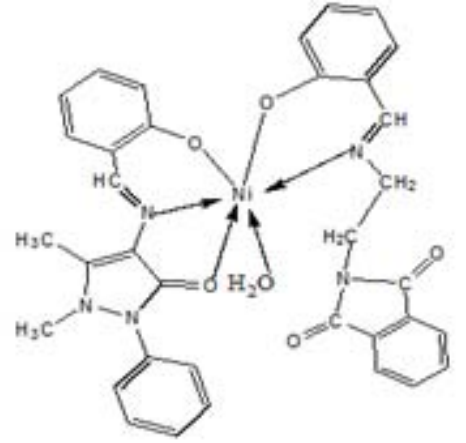

Figure 16: Suggested the structure of $\left[\mathrm{Ni}\left(\mathrm{L}_{1}\right)\left(\mathrm{L}_{2}\right) \mathrm{H}_{2} \mathrm{O}\right]$

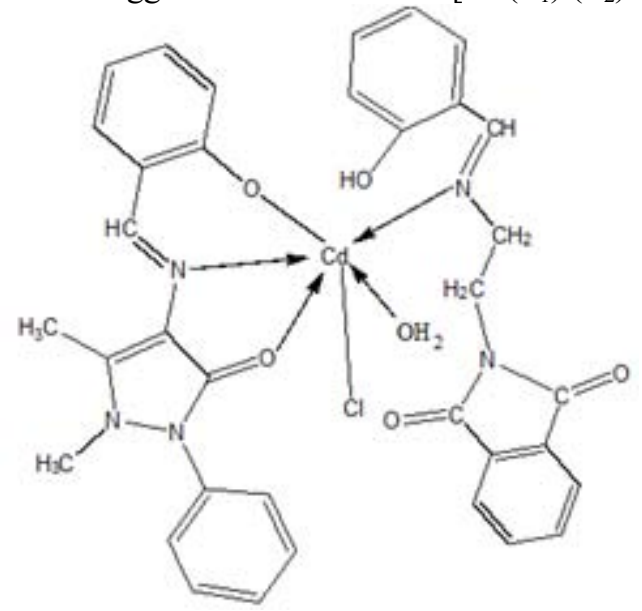

Figure 17: Suggested the structure of $\left[\mathrm{Cd}\left(\mathrm{HL}_{1}\right)\left(\mathrm{L}_{2}\right)\right.$ Cl. $\mathrm{H}_{2} \mathrm{O}$

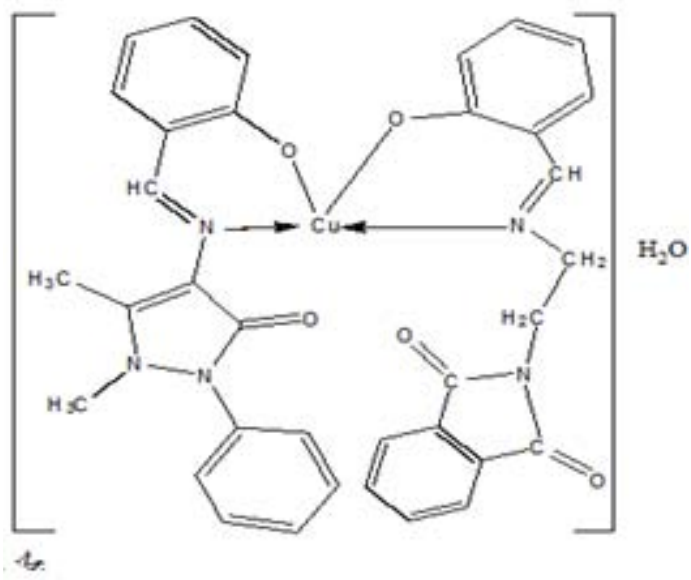

Figure 18: Suggested the structure of $\left.\left[\mathrm{Cu}\left(\mathrm{L}_{1}\right)\left(\mathrm{L}_{2}\right)\right] \mathrm{H}_{2} \mathrm{O}\right]$

\section{Conclusion}

1) From all the data of the infrared spectra of the metal complexes suggested that the metal ion coordinated to the Schiff base ligand through the oxygen atom of phenol 


\section{International Journal of Science and Research (IJSR) \\ ISSN (Online) : 2319-7064}

Index Copernicus Value (2013) : 6.14 | Impact Factor (2014) : 5.611

and the nitrogen atom of the azomethine groups respectively. According to the results characterization of the metal complexes by using different techniques UVVis, C.H.N., atomic absorption (A.A), magnetic moment and molar conductivity have been proposed geometric for complexes are octahedral for[ $\mathrm{Mn}\left(\mathrm{L}_{1}\right)\left(\mathrm{L}_{2}\right) \mathrm{H}_{2} \mathrm{O}$ ], [Ni $\left.\left(\mathrm{L}_{1}\right)\left(\mathrm{L}_{2}\right) \mathrm{H}_{2} \mathrm{O}\right],\left[\mathrm{Cd}\left(\mathrm{HL}_{1}\right)\left(\mathrm{L}_{2}\right) \mathrm{Cl} . \mathrm{H}_{2} \mathrm{O}\right]$ and $[\mathrm{Zn}(\mathrm{HL})$ 2]. $\mathrm{Cl}_{2}$, square planar for $\left[\mathrm{pd}\left(\mathrm{L}_{1}\right)\left(\mathrm{HL}_{2}\right) \mathrm{Cl}\right]$ and $\left[\mathrm{Cu}\left(\mathrm{L}_{1}\right)\right.$ $\left.\left.\left(\mathrm{L}_{2}\right)\right] \mathrm{H}_{2} \mathrm{O}\right]$.

2) The molar conductivity measurements of the complexes were indicated all complexes are non- electrolytic nature.

3) The magnetic measurements were indicated that the above metal $\mathrm{Cu}^{2+}, \mathrm{Mn}^{2+}$ and $\mathrm{Ni}$ complexes are paramagnetic, except the $\mathrm{Pd}^{2+}, \mathrm{Zn}^{2+}$ and $\mathrm{Cd}^{2+}$ complexes are diamagnetic.

\section{References}

[1] Prakash, S., Tuli, G.D., Basu, S.K. and Madan, R.D. (2010)."Advanced inorganic chemistry", $2^{\text {nd }}$ Ed, S. Chand \& company Ltd. PP 3- 40.

[2] Kauffman, C.B. (1981)." Inorganic coordination compounds", Hyden\&Sou Ltd.

[3] Kelode, S.R. (2013)." Synthesis \& Microbial evolution of $\mathrm{VO}$ (IV), $\mathrm{Zr}$ (IV) and $\mathrm{UO}_{2}$ (II) complexes of Schiff base derived from 2-Hydroxy-5-bromo-3nitroacetophenone hydrazide". Int. J. Chem. Pharm. Sci.1 (6) :377-380.

[4] Brown, T. L., Lemary, Jr.H.E., Bursten, B.E., Murphy, C. (2008). "Chemistry: The Central Science", $11^{\text {th }}$ Ed, Prentic Hall.

[5] Yazici, A.; Aydin, H.; Canpolat, E.; and Kaya, M. (2013)." "Synthesis and characterization of two new Schiff Base ligands and their Complexes with some transition metals". J. Chem. Soc.Pak.35 (1) :175-179.

[6] Makode, J.T. and Aswer, A.S. (2004)."Synthesis, characterization, Biological and thermal properties of some newSchiff base complexes derived from 2hydroxy-5-chloro acetophenone and Smethyldithiocarbazate".Indian. J. Chem. 43A: 21202125.

[7] Revanasiddappa, M.; Suresh, T.; Khasim, S.; Radhavendray, S. C.; Basavaraja, C., et al (2008)."Transition metal complexes of1, 4- (2'hydroxyphenyl-1-yl) di-iminoazine: Synthesis, characterization and antimicrobial studies". E. J. Chem. 5:395-403.

[8] Upadhyay, R.K. and Rani, A. (1989).Acta.Chem. Hung. 126-195.

[9] Upadhyay, R. K.; Bajpai, A. K.; and Rathore, K. (1985)."Chemistry of the phenylglyoxal-pdiethylaminoanil and/orthioureaubstituted ammine complexes". Transition metalchemistry 10:24-27.

[10] Ashraf, M.A.; Mahmood, K.; and Wajid, A. (2011)." Synthesis, Characterization and biological activity of Schiffbases". Inter. Conference. Chem. Process.10:1-7.

[11]Kumar, N.; Sharma, P.; and Pareek, A. (2013). "Synthesisof new Schiff base complexesand their applications". I. J. A. R. S. 2 (2) :1-6.

[12] Bheshdadia, B. M., Ph.D.Thesis, (2007)."Studies on metal chelates of some Schiff's bases". University ofSaurashtra, India.
[13] 1-Suresh, M.S. and Prakash, V. (2010). preparaxion and characterizaxion of mexal complexes of schiff base derived from vanillin and 4-aminoantipyrin'; Int.J.Phys.Sci, 5 (14) :2204-2211.

[14] Nakanishi, K. (1962)."Infrared absorption spectroscopy", 1stED, printing in Japan.

[15] Mahmoud, W.A. (1989). "Solution thermodynamics of some uranium complexes". A thesis submitted in fulfillment of the requirement for the D. phid.degree, Sussex university, Sussex university, school of chemistry, and Molecular Science, U.k.

[16] Gebbink, R.J.M.k., Jones, R.J., goldsmith C.R. AND STACK, T.D.R. (2002).Inorg.chem., 41:4633.

[17] Alwan, W.M. (2012). "Synthesis and character zation of azo -linked Schiff base liganedtype (ONO) and its complexes with some metal ions"Athesis submitted to the councid of the college of Education for pure science- Ibn-AL-Haitham of Baghdad University partial fulfillment of the requirements for M.Sc. degree.

[18] Abbas, A.S. (2013). " Metal complexes of Multidentate Schiff base Azo ligand, Syntheses and biological activity ". Dirasat, pure Sci. 39 (1) :61-72.

[19] Lever, A.B.P. (1988). Inorganic spertroscopy. New York. 6: 121.

[20] Schweinfurth D., Krzystek J., Schapiro I., Demeshkosklein J., Telser J., Ozarowski A., yongC., Meyer F., Atanasov M., Neese F. and Sarkar B. (2013). " Electronicstructures of Octahedral Ni (II) complex with click derived triazole ligands; Acombined structural, inagnetometnc spectroscopy and theoretical study.'Inorg.Chem. 52 (12) :6880-6892.

[21]. Dapporto P., Peruzzini M. and stoppioni P. (1981). "Octahedral nickel (II). complexes with the tripod Ligand tris (2-dimethylarsionethyl) amine" inorg.Chem.Acta47:213-216.

[22] Beyeh N.K. and putteddy R. (2015) " Methylresocinarene a reaction vessel to control the coordination geometry of copper (II) complexes Dalton Transaction, 44 (21) :9881-9886.

[23] Matovic Z.D., Miletic v., SamarazicG., Pelosi G., Ianelli S. and Trifunovic S.T. (2015). "Square planer copper (II) complexes with tetradentateamido carboxylate ligands. Crystal structure of $\mathrm{Na}_{2}[\mathrm{Cu}$ (obap) ] ${ }_{2} \mathrm{H}_{2} \mathrm{O}$ strain analysis and spectral assignment of complexsInorg. Chem. Acta.358 (11) :3135-3144.

[24] Trzaskowski B., Les A. and Adamowiczl. (2003) "Modelling of octahedral Manganese (II) complexes with Inorganic ligands:Aproblem with spinstates.'Int.J.mod.Sci, 4:503-511.

[25] TyagiM.and Chandra S. (2014).Synthesis and Spectroscopic studies of biological active tetraazmacroyclic complexes of Mn (II), Co (II), Ni (II), $\mathrm{Pd}$ (II) and pt (II) J.saudi chem..Soc., 18 (1) :53-58.

[26] Oluwafunmilayoadekunle F.A. (2013). "Ni (II), Co (II), Mn (II) and Zn (II) complexes of 5, 6-dihydro-5, 6epoxy-1, 10-Phenanthroline Synthesis and spectroscopic studies. Int. J.Bsic and Appl. Sci (IJBAS-IJENS). 13 (3) :6-10.

[27] Ahmad R.M., Hasan H.A., Yousif E.I. and HussienD.f. (2012). "Synthesis, characterize and biological studies of Cr (III), Mn (II), Zn (II) and cd (II) complex with Schiff base ligand". DiyalaJ.pure Science, 8 (3) : 82100. 


\section{International Journal of Science and Research (IJSR) \\ ISSN (Online) : 2319-7064}

Index Copernicus Value (2013) : 6.14 | Impact Factor (2014) : 5.611

[28] Al-Mogren M.M and Alaghaz A.N. (2013) "Synthesis, Spectral and quantum chemical calculations of mononuclear $\mathrm{Ni}$ (II), $\mathrm{Cu}$ (II), and $\mathrm{Cd}$ (II) complex of new Schiff base ligand"Int.J.electrochem. Soi.8:86698685.

[29] Shit S., Sankolli R. and Gururow T.N. (2014). Adinuclear cadmium (II) Schiff base thiocyanato complex crystal structure and fluorescence".Acta chim.Solv., 61;59-66

[30] Debbeti V., Ahmad T.J., Ananda S. and Gowda N.M. (2012) "Transion metal complexes of ethopropazine :Synthesis and characterization". Amer.J. chem.., 2 (6) :294-298.

[31] Mason W.R. and Gray H. (1968)."Electronic structure of Square planar complexes".j. Amer.Chem..Soc., 90 (21) :5721-5729.

[32]BiswasS., Pramanik, A.K. andMondal T.K. (2015)."Palladium (II) complex with thazolecontaining structure., 1088:28-33.

[33] Seleem H.S. (2011). "Transition metal complexes of an isatinicquinolylhydrazone".chemistry central journal 5 (35) : $1-8$. 